\title{
Compositional methods applied to capital allocation problems
}

\author{
Jaume Belles-Sampera ${ }^{a}$, Montserrat Guillen ${ }^{a}$ and Miguel Santolino ${ }^{a *}$
}

January 20, 2016

\begin{abstract}
In this article we examine the relationship between capital allocation problems and compositional data, i.e., information that refers to the parts of a whole conveying relative information. We show that capital allocation principles can be interpreted as compositions. The natural geometry and vector space structure of compositional data are used to operate with capital allocation solutions. The distance and average that are appropriated in the geometric structure of compositions are presented. We demonstrate that these two concepts can be used to compare capital allocation principles and to merge them. An illustration is provided to show how the distance between capital allocation solutions and the average of these solutions can be computed, and interpreted, by risk managers in practice.
\end{abstract}

JEL classification: C02, G22, D81.

Keywords: Risk management, Simplex, Aitchison distance, Geometric average.

\section{Introduction}

Capital allocation lies on the disaggregation side of the management task, with capital allocation problems arising when a management unit has to distribute a given amount among different agents (where agent should be understood in a broad sense, referring, for instance, to a commercial agent, a business unit, a branch of the main business or a specific guarantee included in a set of contracts). Within financial institutions, capital allocation problems are frequently associated with the fact that risk managers have to determine (at different levels of granularity) agents's contributions to the risk-based capital amount. The company's capital requirement is not the only amount that managers are interested in allocating among agents. Other amounts that can be distributed include the aggregate costs faced by the entity or total retributions. Capital allocation problems are relevant in many other contexts, with similar risk management problems being faced by asset management firms when planning investment strategies or when assessing investment portfolio performance. In these contexts, they are usually referred to as risk attribution or risk budgeting problems (see, for instance, Grégoire, 2007; Rahl, 2012). From the perspective of enterprise risk management (ERM) programs, a sound implementation of capital allocation techniques is primordial to an adequate business analysis.

In this paper we investigate the relationship between capital allocation problems and compositional data analysis. One solution to the capital allocation problem, which depends on an

*Corresponding Author. Department of Econometrics, University of Barcelona, Diagonal 690, 08034Barcelona, Spain. Tel.:+34-93-4020484; fax: +34-93-4021983; e-mail: msantolino@ub.edu 
allocation criterion and a risk measure, is known as the capital allocation principle. We propose that capital allocation principles can be interpreted as compositional data, which constitute quantitative descriptions of the components of a whole, where relative information is more relevant than absolute values. This reinterpretation of capital allocation principles as compositional data enables us to use compositional methods that are coherent with the relative scale of compositions. An immediate practical application of such methods is that capital allocation principles can be ranked according to the distance between them and the associated solutions can be appropriately averaged. Ranking principles can be extremely useful for ordering capital allocation methods and even for deciding which should be used. When decision-makers seek to merge the principles, as opposed to ordering them, computing the mean allocation principle can be valuable. To do so, the simplex and its vector and metric space structures are presented and a discussion is provided as to how we might move from capital allocation principles to compositions, and inversely. The practical application of the compositional methods in the context of capital allocation problems is illustrated with an example extracted from the literature.

A vast number of studies in the literature have been devoted to the analysis of capital allocation problems (see, among others, Denault, 2001; Kalkbrener, 2005; Tsanakas, 2009; Buch et al., 2011; van Gulick et al., 2012). Previous studies have focused on particular probability distributions of losses (Cossette et al., 2012, 2013), on alternative dependence structures between risks (Cai and Wei, 2014), on asymptotic allocations based on commonly used risk measures (Asimit et al., 2011) or on optimization function alterations in order to overcome limitations of the loss function minimization allocation criterion ( $\mathrm{Xu}$ and $\mathrm{Hu}, 2012$; $\mathrm{Xu}$ and $\mathrm{Mao}, 2013$ ). An exhaustive list is not provided here but it is the object of ongoing research. For example, You and Li (2014) analyze capital allocation problems in the context of mutually interdependent risks tied through an Archimedean copula. Wang (2014) investigates the application of the Tail Covariance Premium Adjusted principle in the case of two business lines with exponentially distributed losses, where their dependence structure corresponds to a Farlie-Gumbel-Morgenstern copula. Zaks and Tsanakas (2014) generalize the framework proposed in Dhaene et al. (2012), allowing the inclusion of different hierarchical levels of risk preferences in the final solution. Urbina and Guillén (2014) examine a set of allocation principles to solve capital allocation problems in the context of operational risk. Two interesting recent contributions in this field are Tsanakas and Millossovich (2015) and Li and You (2015). To the best of our knowledge, however, the connection between capital allocation solutions and compositional data has not previously been explored.

The rest of this article is structured as follows. The main concepts of capital allocation problems are presented in the section that follows. The compositional data analysis is introduced in section 3. The connection between capital allocation principles and compositional data is examined in section 4. An illustration of the application of compositional methods is given in section 5. Finally, section 6 concludes.

\section{An overview on capital allocation problems}

A capital allocation problem may be defined as a positive amount $K$ that has to be distributed across $n$ agents in such way that the full allocation condition is satisfied, that is, all $K$ units are 
distributed among the agents. In this framework, a capital allocation principle is the set of $n$ capitals $\left\{K_{i}\right\}_{i=1, \ldots, n}$ such that $\sum_{j=1}^{n} K_{j}=K$. The individual capital $K_{i}$ is the amount assigned to the $i$-th agent, which is computed based on her risk and the risk of the other agents. The agent's risk is represented by a set of random variables $\left\{X_{i}\right\}_{i=1, \ldots, n}$ frequently associated with losses. So, the main elements in a capital allocation problem can be listed as follows:

- The capital $K>0$ to be distributed;

- The agents, indexed by $i=1, \ldots, n$;

- The random variable linked to each agent $X_{i}$;

- A distribution criterion;

- A function $f_{i}$ that concentrates the information of $X_{i}$;

- The capital $K_{i}$ assigned to each agent as a solution to the problem;

- The goal pursued by decision-makers with the allocation principle. Examples include the cost of risk allocation, the reward to risk less allocation or the reward on risk 85 return allocation.

Determining the distribution criterion that drives the allocation is a key issue in the characterization of the capital allocation principle. Here, proportionality to the risk emerges as a natural distribution choice. When a proportional allocation criterion is applied, individual capitals are expressed as a proportion of the aggregate capital, as follows:

$$
K_{i}=K \cdot \frac{f_{i}\left(X_{i}\right)}{\sum_{j=1}^{n} f_{j}\left(X_{j}\right)} \quad, i=1, \ldots, n,
$$

where $f_{i}$ is a function that provides information of the risk $X_{i}$. If the function is a risk measure $\rho\left(X_{i}\right)$, then it is named a stand-alone proportional allocation principle. The criterion that drives the stand-alone proportional allocation principle is that each agent should be assigned the amount of risk that she faces, which is summarized in $\rho\left(X_{i}\right)$ and rescaled by $K$. The main weakness of this principle is that it ignores dependences between risks.

An alternative criterion that takes into account the fact that the capital is distributed among a set of (likely) interdependent agents is that of the marginal proportional allocation principle. Using the notation $S=\sum_{j=1}^{n} X_{j}$, a marginal proportional allocation principle is adhered to if the $f_{i}$ is selected in order to reflect the partial contribution of the loss $X_{i}$ to the value that the risk measure assigns to the aggregate random loss, i.e. $f_{i}=\rho\left(X_{i} \mid S\right)$. It holds, therefore, that $\rho(S)=\sum_{j=1}^{n} \rho\left(X_{j} \mid S\right)$, so the proportional allocation principle based on partial contributions can be expressed as

$$
K_{i}=K \cdot \frac{\rho\left(X_{i} \mid S\right)}{\rho(S)} \quad, i=1, \ldots, n
$$

Non-proportional allocation principles comprise all those solutions in which the individual capitals cannot be expressed as in (1). Many non-proportional principles can be devised when 
using the quadratic optimization criterion in the framework suggested by Dhaene et al. (2012). For instance, non-proportional principles are all the capital allocation principles defined as,

$$
K_{i}=\rho_{i}\left(X_{i}\right)+v_{i} \cdot\left[K-\sum_{j=1}^{n} \rho_{j}\left(X_{j}\right)\right] \quad, i=1, \ldots, n,
$$

where $\rho_{i}$ are risk measures and $v_{i}$ are weights that satisfy $\sum_{j=1}^{n} v_{j}=1$ and at least one of them is not equal to $\rho_{i}\left(X_{i}\right) /\left(\sum_{j=1}^{n} \rho_{j}\left(X_{j}\right)\right)$.

The last element characterizing the capital allocation problem is the specific goal pursued by the decision-makers (e.g., regulators, management team, etc.). The selection of the most suitable capital allocation principle should depend, among other features, on the risk attitudes decision-makers wish to stimulate/penalize among agents. Three possible goals pursued with the capital allocation are cost of risk, reward to risk less and reward on risk 8 return. A capital allocation problem with a cost of risk goal aims to distribute the cost among the agents taking into account some measure of the risk faced by each one of them. An example of the cost of risk goal can be found in the European insurance regulatory framework, Solvency II, where the regulator claims the disaggregation of the solvency capital requirement (SCR) of an insurance company among its lines of business.

If a management team is interested in stimulating a risk-averse attitude among business units, it may adopt a compensation scheme in which rewards are inversely related to the risk level taken by these business units. In such a situation, a capital allocation problem with a reward to risk less objective is promoted. This means, lower risk is associated with higher rewards regardless of returns, and so agents have incentives to take conservative business decisions. Such problems, however, are rare in practice. For instance, it could be used to notionally distribute the contribution of each agent to the overall diversification benefit, where there is only information about a final cost of risk allocation and the overall diversification benefit. More common situations are those in which reward on risk \& return allocations are adhered to in order to reward the performance of agents with higher return/risk ratios. This goal is of great relevance for a sound ERM system because the allocation depends on the relationship between the risk taken and the return obtained. If this is the goal sought then a return-on-risk measure seems to be the natural choice of the $f$ functions. For instance, the return on risk adjusted capital (RORAC) measure, defined as the negative expected value divided by the risk measure value, $\operatorname{RORAC}(X)=-\mathbb{E}(X) / \rho(X)$, could be a suitable candidate. Three different allocation goals are discussed here but other goals might equally be pursued by decision-makers.

\section{Compositional data}

Let's consider the (standard) simplex $\mathcal{S}^{n}=\left\{\vec{z} \in \mathbb{R}^{n} \mid z_{j} \geq 0, j=1, \ldots, n, \sum_{j=1}^{n} z_{j}=1\right\}$ provided with a particular vector and metric space structure. Any vector $\vec{z} \in \mathcal{S}^{n}$ is called a composition and any set of vectors in the simplex is referred to as compositional data. To be considered a vector space over $\mathbb{R}$, a set of vectors needs two operations: vector addition and scalar multiplication. Following the notation used in Aitchison and Egozcue (2005), these two 
operations in the context of compositional data are known as perturbation (denoted by $\oplus$ ) and powering (denoted by $\odot$ ), respectively. The simplex $\mathcal{S}^{n}$ provided with operations $\oplus$ and $\odot$ has a linear vector space structure of dimension $n-1$.

The perturbation operation has to satisfy the following properties: it must be commutative, associative, and have a neutral element and, for each vector, it must be its additive inverse. In the case of the powering operation, the combination of a real number with a vector for a vector space over $\mathbb{R}$ has again to belong to the set of vectors. Additionally, a neutral element of the powering operator must exist. Finally, the distributivity of the perturbation with respect to the powering, and vice versa, must be satisfied. The perturbation and powering operations are defined by expressions (4) and (5), where $\vec{x}, \vec{y} \in \mathcal{S}^{n}$ and $\lambda \in \mathbb{R}$ :

$$
\begin{gathered}
\vec{x} \oplus \vec{y}=\left(\frac{x_{1} \cdot y_{1}}{\sum_{j=1}^{n} x_{j} \cdot y_{j}}, \ldots, \frac{x_{n} \cdot y_{n}}{\sum_{j=1}^{n} x_{j} \cdot y_{j}}\right), \\
\lambda \odot \vec{x}=\left(\frac{x_{1}^{\lambda}}{\sum_{j=1}^{n} x_{j}^{\lambda}}, \ldots, \frac{x_{n}^{\lambda}}{\sum_{j=1}^{n} x_{j}^{\lambda}}\right) .
\end{gathered}
$$

An important concept in compositional data is the closure function, $\mathcal{C}$. The closure function applied to a vector in $\mathbb{R}^{n}$ returns another vector whose components are the components of the original vector divided by the sum of all the components of the original vector. So, the following two expressions hold,

$$
\vec{x} \oplus \vec{y}=\mathcal{C}\left[\left(x_{1} \cdot y_{1}, \ldots, x_{n} \cdot y_{n}\right)\right] \quad, \quad \lambda \odot \vec{x}=\mathcal{C}\left[\left(x_{1}^{\lambda}, \ldots, x_{n}^{\lambda}\right)\right] .
$$

The neutral element of the perturbation in the vector space structure $\left(\mathcal{S}^{n}, \oplus, \odot\right)$ is denoted as $\overrightarrow{0}$. Given a vector $\vec{x}$ such that $x_{i}>0$ for all $i, \vec{r}$ is the inverse of $\vec{x}$ with respect to the perturbation operation if the relationship $\vec{x} \oplus \vec{r}=\overrightarrow{0}$ holds $^{1}$. Therefore, $\vec{r}$ can be expressed as $\vec{r}=(-1) \odot \vec{x}$ or, equivalently, as

$$
\vec{r}=\left(\frac{1 / x_{1}}{\sum_{j=1}^{n}\left(1 / x_{j}\right)}, \ldots, \frac{1 / x_{n}}{\sum_{j=1}^{n}\left(1 / x_{j}\right)}\right) .
$$

Combining this result and (4), we obtain that

$$
\overrightarrow{0}=\vec{x} \oplus \vec{r}=\mathcal{C}\left[\left(\frac{1}{\sum_{j=1}^{n}\left(1 / x_{j}\right)}, \ldots, \frac{1}{\sum_{j=1}^{n}\left(1 / x_{j}\right)}\right)\right]=\left(\frac{1}{n}, \ldots, \frac{1}{n}\right),
$$

which shows that the neutral element $\overrightarrow{0}$ of the perturbation operation is the composition with all elements equal to $1 / n$.

Finally, a distance is required in order to consider the vector space $\left(\mathcal{S}^{n}, \oplus, \odot\right)$ a metric space. Under the simplicial metric defined by Aitchison (1983), the distance between two compositions $\vec{x}$ and $\vec{y}$ is computed as

\footnotetext{
${ }^{1}$ Note that components are strictly positive. The treatment of zeros in compositional data presents some difficulties. See, for instance, Palarea-Albaladejo et al. (2014).
} 


$$
\Delta(\vec{x}, \vec{y})=\|\vec{x} \ominus \vec{y}\|_{\Delta}=\left[\frac{1}{2 n} \sum_{i=1}^{n} \sum_{j=1}^{n}\left[\ln \left(\frac{x_{i}}{x_{j}}\right)-\ln \left(\frac{y_{i}}{y_{j}}\right)\right]^{2}\right]^{1 / 2},
$$

where $\|\cdot\|_{\Delta}$ denotes the norm and $\vec{x} \ominus \vec{y}=\vec{x} \oplus[(-1) \odot \vec{y}]$.

The simplicial arithmetic mean of the compositional data $\vec{x}_{1}, \vec{x}_{2}, \ldots, \vec{x}_{m}$ is computed as,

$$
\mathrm{AM}_{\Delta}\left(\vec{x}_{1}, \ldots, \vec{x}_{m}\right)=\frac{1}{m} \odot \bigoplus_{k=1}^{m} \vec{x}_{k}
$$

where $\bigoplus_{k=1}^{m} \vec{x}_{k}$ means the perturbation of the set of $m$ compositions $\left\{\vec{x}_{k}\right\}_{k=1, \ldots, m}$. As shown in De Baets (2013), this simplicial arithmetic mean may be interpreted as a solution of the minimization problem,

$$
\operatorname{AM}_{\Delta}\left(\vec{x}_{1}, \ldots, \vec{x}_{m}\right)=\arg \min _{\vec{z}} \sum_{k=1}^{m}\left\|\vec{z} \ominus \vec{x}_{k}\right\|_{\Delta}^{2} \quad .
$$

This expression is equivalent to the arithmetic mean of $m$ real numbers $\operatorname{AM}\left(u_{1}, \ldots, u_{m}\right)=$

$\frac{1}{m} \cdot \sum_{k=1}^{m} u_{k}=\arg \min _{v} \sum_{k=1}^{m}\left\|v-u_{k}\right\|_{2}^{2}$. That is, the simplicial metric is an adequate metric when (simplicial) arithmetic means are pursued or, in other words, the expression (7) contains the proper definition of the arithmetic mean of $\vec{x}_{1} \ldots, \vec{x}_{m}$ in the metric space $\left(\mathcal{S}^{n}, \oplus, \odot, \Delta\right)$. An explicit expression for the simplicial arithmetic mean is

$$
\mathrm{AM}_{\Delta}\left(\vec{x}_{1}, \ldots, \vec{x}_{m}\right)=\mathcal{C}\left[\left(G_{1}, \ldots, G_{n}\right)\right],
$$

where $G_{k}=G M\left(x_{1, k}, x_{2, k}, \ldots, x_{m, k}\right), \forall k=1, \ldots, n$. That is, the $G_{k}$ is the geometric mean of $k$-th component of each composition, i.e. $G_{k}=\left[\Pi_{i=1}^{m} x_{i, k}\right]^{1 / m}$. Therefore, the (simplicial) arithmetic mean of the $m$ compositions is computed as the closure of the vector of the geometric means of their components.

\section{From capital allocation principles to compositional data}

We claim that relative capital allocation principles and compositions are naturally linked. Let's denote an (absolute) capital allocation principle by $\vec{K}=\left(K_{1}, K_{2}, \ldots, K_{n}\right)$ and its relative counterpart by the vector $\vec{x}$ with components $x_{i}=K_{i} / K$ satisfying $\sum_{j=1}^{n} x_{j}=1$. An abuse of notation is made because $K$ is used to denote the vector $\vec{K}$ whose components are $K_{i}$ and the amount to be distributed among agents such that $K=\sum_{j=1}^{n} K_{j}$. If our attention is focused on the proportional capital allocation principles defined in expression (1), the relative counterpart $\vec{x}$ of the absolute principle $\vec{K}$ can be interpreted as the closure of the vector with components equal to $f_{i}\left(X_{i}\right), \vec{x}=\mathcal{C}\left[\left(f_{1}\left(X_{1}\right), \ldots, f_{n}\left(X_{n}\right)\right)\right] \forall i=1, \ldots, n$. Similarly, relative non-proportional capital allocation principles can be interpreted as compositions.

Compositional methods can then be used to operate with relative capital allocation principles. For instance, let's assume that a cost of risk relative capital allocation principle $\vec{x}$ is 
implemented and the managers wish to fulfill an allocation with a reward to risk less goal. Intuitively, relative low costs correspond to relative high rewards, so the components should be inverted and the sum normalized in order to provide a full allocation of reward amount $K$. These actions have a natural interpretation in the simplex $\mathcal{S}^{n}$. Given a relative capital allocation principle $\vec{x}$, let $\vec{r}$ be the closure of the vector with components $1 / x_{i}$ for $i=1, \ldots, n$, where $\vec{r}$ is the inverse of $\vec{x}$ with respect to the perturbation operation, $\vec{r}=(-1) \odot \vec{x}$. The application of risk-based capital allocation principles to determine penalizations/rewards may lead to undesirable behaviors on the part of agents. In order to prevent this, the managers may consider direct absolute reward on return \& risk capital allocation principles. Let's assume that the managers allocate rewards based on the RORAC measure and depart from a given $\vec{x}=\mathcal{C}[\vec{y}]$ such that $y_{i}=\operatorname{RORAC}\left(X_{i} \mid S\right) / \operatorname{RORAC}(S)$, where $\operatorname{RORAC}\left(X_{i} \mid S\right)=-\mathbb{E}\left(X_{i}\right) / \rho\left(X_{i} \mid S\right)$ (Tasche, 1999). Then, the absolute principle $\vec{K}$ is obtained by $K_{i}=K \cdot x_{i}, \quad \forall i=1, \ldots, n$. The underlying idea is to provide better rewards to those agents whose relative RORAC with respect to the overall RORAC of the portfolio is highest. Note that alternative definitions of return-on-risk measures may be employed and the objective of the allocation would not change.

One advantage of this relationship between capital allocation principles and compositions is that the geometric structure of the simplex can be used to enrich the description of capital allocation principles. Two important elements to characterize a set of capital allocation principles in the metric space $\left(\mathcal{S}^{n}, \oplus, \odot, \Delta\right)$ are the distance between principles and their average. The simplicial distance $\Delta$ which helps to constitute $\mathcal{S}^{n}$ as a metric space can be useful for comparing capital allocation principles quantitatively. Let's denote $\Delta\left(\vec{x}_{t}, \vec{x}_{g}\right)$ the distance between the relative capital allocation principle $\vec{x}_{t}$ and the relative capital allocation principle $\vec{x}_{g}$. If a relative capital allocation principle is fixed as a benchmark, the distance between this and other relative allocation principles provides a measure of how far they are from the benchmark. By so doing, capital allocation principles can be ranked according to the distance to the capital allocation principle of reference. Of special interest here is the distance between a relative capital allocation principle $\vec{x}$ and $\overrightarrow{0}$. Recall that $\overrightarrow{0} \in \mathcal{S}^{n}$ is the composition in which all $n$ components are equal to $1 / n$, so it can be understood as the neutral capital allocation principle in which all agents are assigned the amount $K / n$. The distance between a relative capital allocation and $\overrightarrow{0}$ provides a quantitative indicator of how far the capital allocation principle is from the neutral assignment.

Finally, the average of a set of capital allocation principles can be computed. In practice, different management teams often provide alternative capital allocations to the same assignment problem. Taking advantage of the geometric structure of $\mathcal{S}^{n}$, the concept of averaging the $m$ points of view on the same allocation problem is easily derived. Let's assume $m$ different relative capital allocation principles $\vec{x}_{k}$ linked to $\vec{K}_{k}, k=1, \ldots, m$. The arithmetic mean of $\vec{x}_{1} \ldots, \vec{x}_{m}$ in the metric space $\left(\mathcal{S}^{n}, \oplus, \odot, \Delta\right)$ is computed as shown in (7). Once the relative arithmetic mean is obtained $\vec{z}$, what remains to be done is to assign an amount of $\bar{K}_{i}=K \cdot z_{i}$ monetary units to each $i$ th agent, $i=1, \ldots, n$, in order to provide an allocation principle that balances the opinions of all the management teams involved. 


\section{Illustration}

To provide a practical illustration of the application, we follow the example given in van Gulick et al. (2012). The authors consider an insurance company offering three types of life insurance portfolios:

- a (deferred) single life annuity that yields a yearly payment in every year that the insured is alive and older than 65 ;

- a survivor annuity that yields a yearly payment in every year that the spouse outlives the insured, if the insured dies before age 65;

- a death benefit insurance that yields a single payment in the year the insured dies, if the insured dies before age 65 ,

with 45, 000 insured males, 15, 000 insured males and 15, 000 insured males, respectively.

The random variables linked to each agent are the present values of liability payments made to the insureds in each portfolio, and are represented by $X_{s l}, X_{\text {surv }}$ and $X_{d b}$, respectively. The capital amount to be distributed among the agents is $K=\operatorname{TVaR}_{99 \%}(S)$, being $S=X_{s l}+X_{s u r v}+X_{d b}$. Three capital allocation principles are studied in van Gulick et al. (2012): two proportional allocation criteria and one non-proportional criterion ${ }^{2}$. The two proportional allocation criteria consist of a stand-alone proportional allocation principle, based on the standard deviation as risk measure, and a marginal proportional allocation principle, where partial contributions are computed in accordance with the gradient allocation principle ${ }^{3}$. Finally, the authors developed a non-proportional allocation that lexicographically minimizes a portfolio's excesses, denominated the excess based allocation principle. We use the symbols $\sigma, \nabla$, and EBA to denote the proportional principle based on the standard deviation, the gradient allocation principle and the excess based allocation principle, respectively.

The results provided in van Gulick et al. (2012) are as follows: the amount to be allocated is equal to $K=\mathrm{TVaR}_{99 \%}\left(X_{s l}+X_{\text {surv }}+X_{d b}\right)=376,356$, and the allocations derived from the principles are shown in Table 1 . The relative capital allocation principles associated with the absolute principles, which belong to the simplex $\mathcal{S}^{3}$, are also presented.

These three capital allocation principles are driven by a cost of risk goal. The inverse of these principles can be computed. Let's assume that risk managers are interested in the assignment of diversification benefits among agents based on an allocation driven by a reward to risk less objective. To move from the cost of risk goal to the reward to risk less objective, the inverse of the relative capital allocation principle with respect to the perturbation operation $\vec{r}$ is computed and the absolute allocations are derived. The risk measure values are $\operatorname{TVaR}_{99 \%}\left(X_{s l}\right)=364,477$, $\operatorname{TVaR}_{99 \%}\left(X_{\text {surv }}\right)=11,657$ and $\operatorname{TVaR}_{99 \%}\left(X_{d b}\right)=6,346$, so the diversification benefit to share among agents is $(364,477+11,657+6,346)-376,356=382,480-376,356=6,124$. Results

\footnotetext{
${ }^{2}$ The authors analyzed an additional capital allocation principle that is omitted here because it reported negative allocations.

${ }^{3}$ The gradient allocation principle is also known as the Euler allocation principle (McNeil et al., 2005) or, from the game-theoretic perspective, the Aumann-Shapley allocation principle (Denault, 2001).
} 
Table 1: Cost of risk based capital allocation principles to allocate the aggregate risk and their relative counterparts

\begin{tabular}{lccc} 
& $\begin{array}{l}\text { Single } \\
\text { annuity } \\
\left(X_{s l}\right)\end{array}$ & $\begin{array}{l}\text { life } \\
\text { annuity } \\
\left(X_{\text {surv }}\right)\end{array}$ & $\begin{array}{c}\text { Death } \\
\text { benefit } \\
\left(X_{d b}\right)\end{array}$ \\
\hline Absolute allocations \\
Proportional principle based on st.dev. $(\sigma)$ & 335,724 & 24,725 & 15,907 \\
Gradient allocation principle $(\nabla)$ & 364,477 & 7,979 & 3,900 \\
Excess based allocation principle (EBA) & 360,324 & 10,495 & 5,537 \\
& \multicolumn{3}{c}{ Relative allocations } \\
Proportional principle based on st.dev. $(\sigma)$ & $89.20 \%$ & $6.57 \%$ & $4.23 \%$ \\
Gradient allocation principle $(\nabla)$ & $96.84 \%$ & $2.12 \%$ & $1.04 \%$ \\
Excess based allocation principle $(\mathrm{EBA})$ & $95.74 \%$ & $2.79 \%$ & $1.47 \%$ \\
\hline The aggregate risk is $K=\mathrm{TVR}_{99 \%}(S)=376,356$. & Information provided in van Gulick et al. $(2012)$.
\end{tabular}

are shown in Table 2.

We should emphasize that the allocations shown in Table 2 do not directly match the allocations derived from individual diversification benefits. For example, individual diversification benefits linked to the gradient allocation principle are equal to 0 for the single life annuity $(364,477$ $364,477), 3,678$ for the survivor annuity $(11,657-7,979)$ and 2,446 for the death benefit $(6,346-$ $3,900)$. These values may be interpreted as an absolute allocation principle directly derived from the aggregate diversification benefit, with the relative counterpart $(0.00 \%, 60.06 \%, 39.94 \%)$. In contrast, the values of the relative gradient allocation principle shown in Table 2 are, respectively, $0.71 \%, 32.60 \%$ and $66.69 \%$. These differences reflect the fact that the former is based on the absolute diversification benefit of each agent, while the latter takes into account the relative riskiness of each agent with respect to the rest of the agents.

These relative principles can be ranked in accordance with their simplicial distances. Imagine that decision-makers wish to know the distances between these allocation principles and the neutral allocation principle -denoted as $\overrightarrow{0}$-, and the distances between the proportional principle based on the standard deviation and the excess based allocation principle with the gradient allocation principle, respectively. Simplicial distances may be calculated from expression (6), as follows:

$$
\begin{gathered}
\Delta_{\sigma, \overrightarrow{0}}=\sqrt{3.557+0.522+1.354}=2.3308, \\
\Delta_{\nabla, \overrightarrow{0}}=\sqrt{7.764+1.072+3.066}=3.4499, \\
\Delta_{\mathrm{EBA}, \overrightarrow{0}}=\sqrt{6.608+0.932+2.576}=3.1806,
\end{gathered}
$$

and

$$
\Delta_{\sigma, \nabla}=\sqrt{0.8107+0.0978+0.3452}=1.1197
$$


Table 2: Reward to risk less based capital allocation principle to allocate the diversification benefits and their relative counterparts

\begin{tabular}{|c|c|c|c|}
\hline & $\begin{array}{l}\text { Single life } \\
\text { annuity } \\
\left(X_{s l}\right)\end{array}$ & $\begin{array}{l}\text { Survivor } \\
\text { annuity } \\
\left(X_{\text {surv }}\right)\end{array}$ & $\begin{array}{l}\text { Death } \\
\text { benefit } \\
\left(X_{d b}\right)\end{array}$ \\
\hline & \multicolumn{3}{|c|}{ Absolute allocations ${ }^{*}$} \\
\hline Proportional principle based on st.dev. $(\sigma)$ & 172 & 2,330 & 3,622 \\
\hline Gradient allocation principle $(\nabla)$ & 44 & 1,996 & 4,084 \\
\hline \multirow[t]{2}{*}{ Excess based allocation principle (EBA) } & 61 & 2,094 & 3,969 \\
\hline & \multicolumn{3}{|c|}{ Relative allocations } \\
\hline Proportional principle based on st.dev. $(\sigma)$ & $2.80 \%$ & $38.05 \%$ & $59.15 \%$ \\
\hline Gradient allocation principle $(\nabla)$ & $0.71 \%$ & $32.60 \%$ & $66.69 \%$ \\
\hline Excess based allocation principle (EBA) & $1.00 \%$ & $34.19 \%$ & $64.81 \%$ \\
\hline
\end{tabular}

* The aggregate diversification benefit is equal to 6,124 .

$$
\Delta_{\mathrm{EBA}, \nabla}=\sqrt{0.0466+0.0049+0.0213}=0.2698
$$

Distances taken individually are normally not especially informative. However, a comparison of the distances can be used to rank the principles. In Figure 5 the simplicial distances of the relative principles with respect to the neutral relative principle are shown. In this example, the gradient allocation principle provides the furthest allocation in comparison to the neutral allocation, followed by the EBA and the $\sigma$ allocations. If gradient allocation is the principle of reference, then the EBA allocation principle is closer than the $\sigma$ principle.

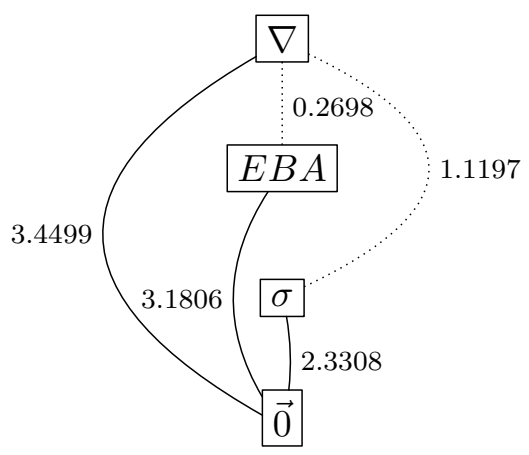

Figure 1: Simplicial distances between capital allocation principles. Solid lines represent the distances of principles against the neutral allocation principle $(\overrightarrow{0})$. Dotted lines represent the distances of principles against the gradient principle $(\nabla)$. 
Finally, the average of the three relative allocations is computed. Let $\vec{x}_{\sigma}, \vec{x}_{\nabla}$ and $\vec{x}_{\mathrm{EBA}}$ denote each of the three relative allocations displayed in Table 1, that is,

$$
\begin{aligned}
& \vec{x}_{\sigma}=(89.20 \%, 6.57 \%, 4.23 \%), \\
& \vec{x}_{\nabla}=(96.84 \%, 2.12 \%, 1.04 \%) \text { and } \\
& \vec{x}_{\text {EBA }}=(95.74 \%, 2.79 \%, 1.47 \%) .
\end{aligned}
$$

To average these principles, first, the three geometric means of the three components of these relative allocations are computed. Their values are

$$
\begin{aligned}
& G_{1}=(89.20 \% \cdot 96.84 \% \cdot 95.74 \%)^{1 / 3}=93.87 \%, \\
& G_{2}=(6.57 \% \cdot 2.12 \% \cdot 2.79 \%)^{1 / 3}=3.39 \% \text { and } \\
& G_{3}=(4.23 \% \cdot 1.04 \% \cdot 1.47 \%)^{1 / 3}=1.88 \% .
\end{aligned}
$$

Following expression (8), the simplicial arithmetic mean $\mathrm{AM}_{\Delta}\left(\vec{x}_{\sigma}, \vec{x}_{\nabla}, \vec{x}_{\mathrm{EBA}}\right)$ is obtained as the closure of the vector with components equal to the geometric means $\mathcal{C}\left[\left(G_{1}, G_{2}, G_{3}\right)\right]$. The simplicial average of relative principles and the associated absolute allocation are shown in Table 3. Note that the simplicial mean is the adequate average in the simplex $\mathcal{S}^{3}$. The values shown in Table 3 differ from the arithmetic mean of the components of the original relative principles, which are, respectively, $93.93 \%, 3.83 \%$ and $2.25 \%$.

Table 3: Average of the three capital allocation principles

\begin{tabular}{llll} 
& $\begin{array}{l}\text { Single life } \\
\text { annuity } \\
\left(X_{s l}\right)\end{array}$ & $\begin{array}{l}\text { Survivor } \\
\text { annuity } \\
\left(X_{\text {surv }}\right)\end{array}$ & $\begin{array}{l}\text { Death } \\
\text { benefit } \\
\left(X_{d b}\right)\end{array}$ \\
\hline Average (absolute allocations) & 356,431 & 12,859 & 7,066 \\
$A M_{\Delta}$ (relative allocations) & $94.71 \%$ & $3.42 \%$ & $1.88 \%$ \\
\hline
\end{tabular}

\section{Conclusions}

The relationship between capital allocation problems and compositional data has been demonstrated, as has the fact that relative capital allocation principles can be interpreted as compositions. The immediate implication of this is that compositional methods can be applied to capital allocation solutions. In the foregoing discussion, we have focused on the simplicial distance and the simplicial arithmetic mean and on the information provided by them when applied to capital allocation solutions. We show that the simplicial distance is useful for ranking allocations based on distances and argue that managers can compute these distances to measure just how far alternative allocation solutions lie from the neutral allocation solution in which all agents are assigned the same amount. However, it should be noted that the arithmetic mean cannot be used directly to average capital allocation solutions. Rather, the simplicial arithmetic mean needs to be computed if decision-makers hope to obtain an adequate average of a set of allocation solutions. 
In this study we have explored the information provided by a limited number of compositional methods when applied to allocation solutions. As such, the aim has been to clarify the relationship between these two areas of research. However, there are many other elements involved in compositional data analysis and their application to allocation solutions deserves the attention of our future research. It is our firm belief that there remains considerable scope for further research connecting these fields.

\section{Acknowledgements}

The authors acknowledge the support of the Spanish Ministry for grants ECO2013-48326-C2-1-P and ECO2012-35584. MG thanks ICREA Academia.

\section{Declaration of interests}

The authors report no conflicts of interest. The authors alone are responsible for the content and writing of the paper 


\section{References}

J. Aitchison. Principal component analysis of compositional data. Biometrika, 70(1):57-65, 1983.

J. Aitchison and J. Egozcue. Compositional data analysis: Where are we and where should we be heading? Mathematical Geology, 37(7):829-850, 2005.

A. V. Asimit, E. Furman, Q. Tang, and R. Vernic. Asymptotics for risk capital allocations based on Conditional Tail Expectation. Insurance: Mathematics and Economics, 49(3):310324, 2011. ISSN 0167-6687. doi: 10.1016/j.insmatheco.2011.05.002.

A. Buch, G. Dorfleitner, and M. Wimmer. Risk capital allocation for RORAC optimization. Journal of Banking and Finance, 35(11):3001-3009, 2011.

J. Cai and W. Wei. Some new notions of dependence with applications in optimal allocations problems. Insurance: Mathematics and Economics, 55:200-209, 2014.

H. Cossette, M. Mailhot, and E. Marceau. TVaR-based capital allocation for multivariate compound distributions with positive continuous claim amounts. Insurance: Mathematics and Economics, 50:247-256, 2012.

H. Cossette, M. Côté, E. Marceau, and K. Moutanabbir. Multivariate distribution defined with Farlie-Gumbel-Morgenstern copula and mixed Erland marginals: Aggregation and capital allocation. Insurance: Mathematics and Economics, 52:560-572, 2013.

B. De Baets. Aggregation 2.0. Opening plenary session of the AGOP 2013 conference, Pamplona, Spain, 2013.

M. Denault. Coherent allocation of risk capital. Journal of Risk, 4(1):1-34, 2001.

J. Dhaene, A. Tsanakas, E. A. Valdez, and S. Vanduffel. Optimal capital allocation principles. Journal of Risk and Insurance, 79(1):1-28, 2012. ISSN 1539-6975. doi: 10.1111/j.15396975.2011.01408.x.

P. Grégoire. Advanced Portfolio Attribution Analysis: New Approaches to Return and Risk, chapter 12 - Risk Attribution, pages 309-329. Riskbooks, Incisive Media, 2007.

M. Kalkbrener. An axiomatic approach to capital allocation. Mathematical Finance, 15(3): 425-437, 2005. ISSN 1467-9965. doi: 10.1111/j.1467-9965.2005.00227.x.

X. Li and Y. You. Permutation monotone functions of random vectors with applications in financial and actuarial risk management. Advances in Applied Probability, 47(1):270-291, 2015 .

A. J. McNeil, R. Frey, and P. Embrechts. Quantitative Risk Management. Princeton Series in Finance. Princeton University Press, New York, 2005.

J. Palarea-Albaladejo, J. Martín-Fernández, and R. Olea. Bootstrap estimation of distributional statistics from compositional data with nondetects: a case study on coal ashes. Journal of Chemometrics, 2(28):585-599, 2014. 
L. Rahl, editor. Risk Budgeting: Risk Appetite and Governance in the Wake of the Financial Crisis. Riskbooks, Incisive Media, 2nd edition, 2012.

D. Tasche. Risk contributions and performance measurement. Working paper, Lehrstuhl für Mathematische Statistik, TU München, 1999.

A. Tsanakas. To split or not to split: Capital allocation with convex risk measures. Insurance: Mathematics and Economics, 44(2):268-277, 2009. ISSN 0167-6687. doi: 10.1016/j.insmatheco.2008.03.007.

A. Tsanakas and P. Millossovich. Sensitivity analysis using risk measures. Article, Cass Business School, City University London, 2015.

J. Urbina and M. Guillén. An application of capital allocation principles to operational risk and the cost of fraud. Expert Systems with Applications, 41(16):7023-7031, 2014.

G. van Gulick, A. De Waegenaere, and H. Norde. Excess based allocation of risk capital. Insurance: Mathematics and Economics, 50(1):26-42, 2012. ISSN 0167-6687. doi: 10.1016/j.insmatheco.2011.09.003.

M. Wang. Capital allocation based on the tail covariance premium adjusted. Insurance: Mathematics and Economics, 57:125-131, 2014.

M. Xu and T. Hu. Stochastic comparisons of capital allocations with applications. Insurance: Mathematics and Economics, 50(3):293-298, 2012. ISSN 0167-6687. doi: 10.1016/j.insmatheco.2011.12.004.

M. Xu and T. Mao. Optimal capital allocation based on the Tail Mean-Variance model. Insurance: Mathematics and Economics, 53:533-543, 2013.

Y. You and X. Li. Optimal capital allocations to interdependent actuarial risks. Insurance: Mathematics and Economics, 57:104-113, 2014.

Y. Zaks and A. Tsanakas. Optimal capital allocation in a hierarchical corporate structure. Insurance: Mathematics and Economics, 56:48-55, 2014. 